\title{
CARACTERÍSTICAS EPIDEMIOLÓGICAS DE PACIENTES HOSPITALIZADOS COM INFARTO AGUDO DO MIOCÁRDIO EM BELÉM-PA
}

\section{EPIDEMIOLOGICAL CHARACTERISTICS OF HOSPITALIZED PATIENTS WITH ACUTE MYOCARDIAL INFARCTION IN BELÉM-PA}

Ana Carolina Cancela Alvarez ${ }^{1}$, Ramon William da Silva Rezende ${ }^{2}$, Emanuel de Jesus Soares de Sousa ${ }^{3}$.

\section{RESUMO}

Dentro do cenário de saúde coletiva mundial e nacional, as doenças cardiovasculares (DCV) estão entre as principais causa de mortalidade e morbidade. O Infarto Agudo do Miocárdio, por sua vez, caracteriza-se como o principal expoente dentre todas as DCV's, em prevalência. O presente estudo objetivou descrever o perfil epidemiológico dos pacientes hospitalizados por IAM em Belém do Pará, entre janeiro de 2016 a novembro de 2020. Os resultados revelaram um total de 5.854 pacientes internados por IAM em Belém do Pará entre 2016 a 2020. Dentre as características epidemiológicas avaliadas, notou-se que a maioria foram do sexo masculino $(70,8 \%)$, pardos $(80 \%)$ e idosos $(62,29 \%)$. Apesar de ter sido notado uma queda vultuosa nos números de óbitos dos pacientes hospitalizados por IAM, sobretudo em 2020, não foi possível associar esse resultado a melhorias do manejo intensivo e da infraestrutura hospitalar. Ao que parece, semelhante a outros estudos, a queda do número de óbitos durante o momento supracitado teve influência direta da pandemia do SARS-CoV2/COVID-19. Em 2020, a maioria das internações em centros intensivos foram de pacientes com síndrome respiratória aguda. Nesse sentido, notou-se a diminuição do número de internações por outras doenças devido à escassez de leitos.

Palavras-chave: Epidemiologia. Cardiologia. Infarto do Miocárdio.

\section{ABSTRACT}

Within the global and national collective health scenario, cardiovascular diseases (CVD) are among the main causes of mortality and morbidity. Acute Myocardial Infarction, in turn, is characterized as the main exponent among all CVD's, in prevalence. The present study aimed to describe the epidemiological profile of patients hospitalized for AMI in Belém do Pará, between January 2016 and November 2020. The results revealed a total of 5,854 patients hospitalized for AMI in Belém do Pará between 2016 and 2020. Among the characteristics assessed, it was noted that the majority were male $(70.8 \%)$, brown $(80 \%)$ and elderly $(62.29 \%)$. Although a large drop in the number of deaths of patients hospitalized for AMI was noted, especially in 2020, it was not possible to associate this result with improvements in intensive management and hospital infrastructure. Apparently, similar to other studies, the drop in the number of deaths during the aforementioned moment had a direct influence on the SARS-CoV2 / COVID-19 pandemic. In 2020, most hospitalizations in intensive care units were for patients with acute respiratory syndrome. In this sense, there was a decrease in the number of hospitalizations for other diseases due to the shortage of beds.

Keywords: Epidemiology; Cardiology; Myocardial Infarction.
Graduanda em Medicina na Universidade Estadual do Pará.

E-mail:

anacarolinamed2022@gmail.com

Graduando em Medicina na Universidade Estadual do Pará.

Graduado em Medicina na Universidade Federal do Pará 


\section{INTRODUÇÄO}

Dentro do cenário de saúde coletiva mundial e nacional, as doenças cardiovasculares (DCV) estão entre as principais causa de mortalidade e morbidade. O Infarto Agudo do Miocárdio, por sua vez, caracteriza-se como o principal expoente dentre todas as DCV's, em prevalência. Essa patologia se dá pela morte celular da musculatura cardíaca secundária a um quadro de má perfusão tecidual, normalmente com uma etiologia isquêmica que compromete a irrigação coronária em uma determinada topografia do coração. ${ }^{1-2-3-4}$

Diversos são os estudos que analisam os principais fatores relacionados ao aumento da prevalência de IAM nas populações. Maus hábitos alimentares, estresse emocional, sedentarismo, tabagismo, obesidade e fatores socioeconômicos estão relacionados com o aumento da manifestação de IAM. O aumento da expectativa de vida global também é visto como um significativo contribuinte para o elevar das estatísticas envolvendo DCV. ${ }^{5-6}$

Em uma pesquisa realizada para avaliar a epidemiologia das DCV's nos países de língua portuguesa, notou-se correlações relevantes. As condições socioeconômicas dos países analisados influenciaram diretamente nas estatísticas. Nessa perspectiva, notouse que as nações africanas analisadas apresentaram maiores prevalências de doenças cerebrovasculares do que cardiovasculares. Entretanto, dentre as DCV's, as do tipo isquêmicas estiveram associadas aos maiores números de óbitos. Ressalta-se, também, que os fatores de risco mais relevantes para todas as DCV's nos países de língua portuguesa estudados foram hipertensão arterial e fatores dietéticos. ${ }^{7}$

No estado do Pará, nota-se um aumento do número de internações por IAM nos últimos anos. Em contra partida, verifica-se uma diminuição na proporção de óbitos desses pacientes hospitalizados. É válido ressaltar que as subnotificações, sobretudo na região norte do país, dificultam a construção de estatísticas clínicas e epidemiológicas fidedignas ao cenário de saúde coletiva. ${ }^{8}$

Classicamente, o quadro clínico do IAM surge com uma dor torácica típica de síndrome coronariana aguda (SCA). As características de dor em aperto, queimação, pressão ou peso associadas, ou não, a irradiação para membro superior, mandíbula juntamente com desconforto em epigastro- podem estar presentes em uma SCA. Os traçados eletrocardiográficos e marcadores séricos de lesão miocárdica -sobretudo as troponinas- ajudam a confirmar o caso e auxiliar no manejo do IAM. ${ }^{9}$ 
O manejo correto do paciente com IAM está diretamente relacionado com a queda da curva de óbitos relacionados a essa injúria. A demora na identificação e intervenção do quadro mostram estar associados aos piores desfechos ao paciente. Verifica-se a importância dos centros hospitalares na captação da demanda dos pacientes diagnosticados com IAM, sobretudo os serviços de referência cardiológicos que possuem centro de hemodinâmica e profissionais especializados. No geral, todos esses aspectos implicam em uma redução da morbimortalidade relacionada ao IAM. ${ }^{10}$

Tendo em vista o cenário acima, percebe-se a grande relevância clínica e epidemiológica do IAM no Brasil. Entretanto, verifica-se uma quantidade insuficiente de trabalhos voltados para a caracterização epidemiológica do IAM na região norte do país. Dessa forma, o presente estudo objetivou descrever o perfil epidemiológico dos pacientes hospitalizados por IAM em Belém do Pará, entre janeiro de 2016 a novembro de 2020. Justifica-se tal período pelo fato de o mesmo refletir o momento epidemiológico atual de forma mais fidedigna.

\section{MATERIAIS E METODOS}

Foi realizado um estudo retrospectivo, quantitativo do tipo ecológico a partir da coleta de dados presentes no Sistema de Informações Hospitalares/Departamento de Informática do Sistema Único de Saúde (SIH/DATASUS) do Ministério da Saúde através da plataforma DATASUS/TABNET.

Os dados utilizados foram de pacientes hospitalizados por Infarto Agudo do Miocárdio (CID 1219) durante o período de janeiro de 2016 a novembro de 2020. As variáveis epidemiológicas selecionadas foram: número de internações, número de óbitos, sexo, etnia e faixa etária. Os dados foram coletados a partir da plataforma SIH/DATASUS/TABNET, e as plataformas TABWIN/DATASUS, Word 2013 e Excel 2013 foram usadas para construir as análises e representações gráficas.

É importante destacar que por ter ocorrido coleta de dados classificados como secundários e não ter acontecido abordagens diretas a pessoas e prontuários, o parecer institucional do Comitê de Ética em Pesquisa institucional não foi necessário. Entretanto, todos os preceitos éticos do Código de Nuremberg e da Declaração de Helsinque foram adotados para a elaboração deste estudo. 


\section{RESULTADOS E DISCUSSÃO}

Em valor absoluto, entre os anos de 2016 a 2020, o presente estudo verificou um total de 5.854 pacientes que foram hospitalizados por infarto agudo do miocárdio em Belém do Pará. Em relação as internações por ano, tem-se 1.122, 1.223, 1.408, 1.167 e 934 referentes aos anos de 2016, 2017, 2018, 2019 e 2020, conforme demonstrado na figura 1. É possível perceber que dos anos de 2016 a 2019 os números de internações se mantiveram praticamente constantes, com poucas variações. Essa verificação mostra a grande relevância epidemiológica do IAM, visto que dentre as DCV's é a que detém a maior prevalência global e influencia diretamente nos aspectos de morbimortalidade mundial. ${ }^{11}$

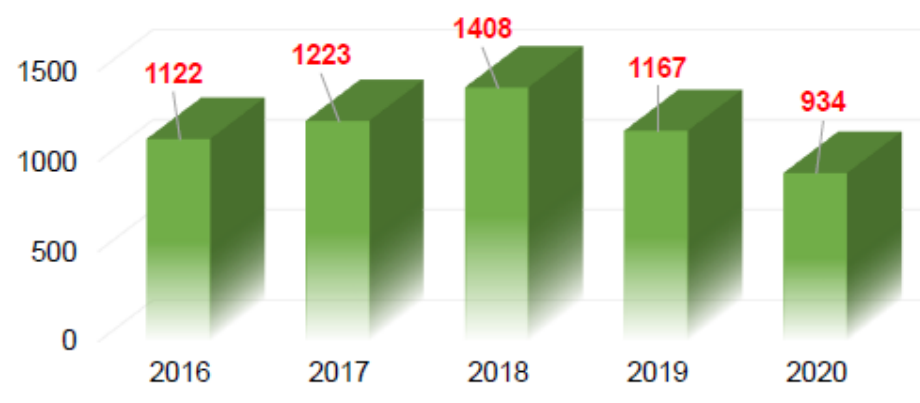

Figura 1: Representação do número de casos de pacientes hospitalizados por IAM no o período de 2016 a 2020, em Belém do Pará.

Fonte: Ministério da Saúde. SIH/SUS. 2021.

No ano de 2020, notou-se que o número absoluto de internações teve uma queda singular em relação aos demais anos analisados. Possivelmente, o momento de pandemia do SARS-CoV-2/COVID-19 influenciou diretamente no número de internações. Aparentemente, as subnotificações epidemiológicas não foram as principais responsáveis pelo contrassenso. Mascarenhas ${ }^{12}$ aponta para essa situação, pois concorda que a estrutura precária do sistema de saúde brasileiro, não teve capacidade de comportar todas as demandas emergenciais e, por isso, o número de leitos de tratamento intensivo estavam ocupados com pacientes que estavam com a síndrome respiratória do COVID19. ${ }^{13-14-15}$

Entre 2016 a 2020, o número de homens foi superior ao das mulheres em relação às internações por IAM: $4.146(70,8 \%)$ e 1.708 (29,2\%), como pode ser verificado na figura 
DOI: 10.18606/2318-1419/amazonia.sci.health.v9n1p69-78
ALVAREZ, ACC. REZENDE, RWS. SOUSA, EJS.

Características Epidemiológicas de Pacientes Hospitalizados por Infarto Agudo do Miocárdio em Belém do Pará.

2. Dentre as hipóteses presentes na literatura para que ocorra esta desproporção estão, justamente, uma maior prevalência de maus hábitos de vida - dietético e sedentarismo, principalmente - entre o sexo masculino; bem como erros diagnósticos no que diz respeito a identificação imediata da SCA incluindo seu manejo adequado. Contudo, alguns estudos notabilizam que a tendência global atualmente é de que o número de mulheres que desenvolvem a síndrome coronariana aguda e evoluem para o infarto agudo do miocárdio está em uma crescente. ${ }^{16-17}$

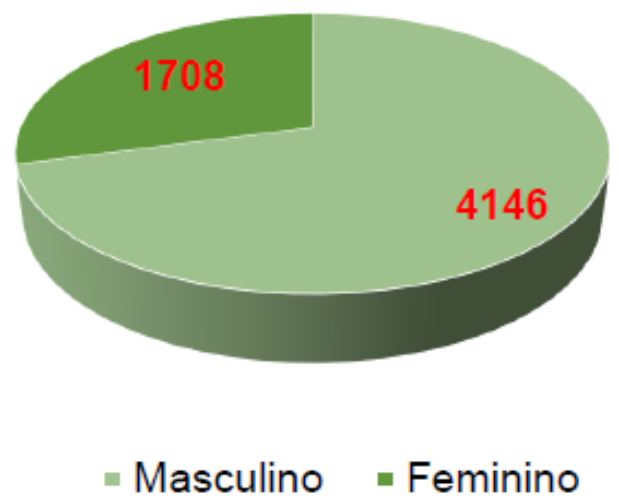

Figura 2: Representação do sexo dos pacientes hospitalizados por IAM durante o período de 2016 a 2020, em Belém do Pará.

Fonte: Ministério da Saúde. SIH/SUS. 2021.

Os indivíduos idosos tiveram o maior número de internações durante o período analisado, sendo $62,29 \%$ de acordo com o quadro 1. Esse dado concorda com diversos estudos que verificam que há uma relação proporcional direta entre o avançar da idade e o aumento da prevalência de IAM. Não somente os fatores modificáveis, como dieta e exercício físico, mas principalmente os fatores não modificáveis, como as doenças crônicas, que mostram ser as mais importantes variáveis influenciadoras do alavancar das estatísticas de IAM na população com mais de 60 anos. ${ }^{18}$

Quadro 1: Disposição da faixa etária dos pacientes hospitalizados por IAM durante o período de 2016 a 2020, em Belém do Pará.

\begin{tabular}{|l|c|c|c|c|}
\hline VARIÁVEL & - & № & $\%$ & P-Valor \\
\hline \multirow{3}{*}{ Faixa Etária } & $0-18$ Anos & 6 & $0,1 \%$ & \multirow{2}{*}{$\mathrm{P}<0,0001$} \\
\cline { 2 - 4 } & $19-59$ Anos & 2.201 & $37,59 \%$ & \\
\cline { 2 - 4 } & & 3.647 & $62,29 \%$ & \\
\hline
\end{tabular}

Fonte: Ministério da Saúde. SIH/SUS. 2021. 
DOI: 10.18606/2318-1419/amazonia.sci.health.v9n1p69-78
ALVAREZ, ACC. REZENDE, RWS. SOUSA, EJS.

Características Epidemiológicas de Pacientes Hospitalizados por Infarto Agudo do Miocárdio em Belém do Pará.

O grupo de indivíduos adultos hospitalizados com IAM, em Belém do Pará, também obteve valores elevados que, por sua vez, totalizaram $37,59 \%$ dos casos. É possível associar esse resultado com achados de outros estudos semelhantes que atestam uma crescente na incidência de IAM na população adulta no geral. Alguns resultados que tentam explicar essa crescente apontam que dentre os fatores de riscos mais frequentemente associados à injúria cardíaca, tem-se que o abuso de substâncias ilícitas - como derivados da cocaína - e uma rotina dietética baseada em alto consumo calórico estão constantemente presentes entre os adultos. ${ }^{19}$

O quadro 2 revela que os pacientes da cor/raça parda foram os que tiveram as maiores prevalências de IAM do que as demais, somando $80 \%$ do total de casos. Muitos estudos atestam que a população negra e parda detém maior propensão a desenvolver doenças cardiovasculares do que as demais raças. Entretanto, esse significativo resultado de $80 \%$ entre os pardos pode ser um viés, justamente pelo fato de existir uma grande influência da construção histórica, social e cultural de Belém do Pará neste resultado. Salienta-se que a maioria da população da capital paraense é composta por autodeclarados pardos e negros. ${ }^{8,20}$

Quadro 2: Etnia dos pacientes hospitalizados com IAM durante o período de 2016 a 2020, em Belém do Pará.

\begin{tabular}{|l|l|l|l|l|}
\hline VARIÁVEL & - & $\mathbf{N}^{\mathbf{0}}$ & $\%$ & P-Valor \\
\hline \multirow{4}{*}{ Etnia } & Branca & 208 & $3,5 \%$ & \\
\cline { 2 - 4 } & Preta & 96 & $1,6 \%$ \\
\cline { 2 - 4 } & Parda & 4.686 & $80 \%$ & \multirow{3}{*}{$\mathrm{P}<0,0001$} \\
\cline { 2 - 4 } & Amarela & 0 & $0,0 \%$ \\
\cline { 2 - 4 } & Indígena & 0 & $0,0 \%$ \\
\cline { 2 - 4 } & Sem informação & 864 & $14,7 \%$ & \\
\hline
\end{tabular}

Fonte: Ministério da Saúde. SIH/SUS. 2021.

Estudos brasileiros demonstram um aumento progressivo dos óbitos relacionados ao IAM nos últimos anos no Brasil. 8,17,20,21 Entretanto, de acordo com a figura 3, notou-se que durante o período estudado, na cidade de Belém-PA, houve uma relativa constante no número de óbitos entre 2016 a 2018, mas que cursou com uma queda significativa destes valores nos anos de 2019 e 2020. 
DOI: 10.18606/2318-1419/amazonia.sci.health.v9n1p69-78
ALVAREZ, ACC. REZENDE, RWS. SOUSA, EJS.

Características Epidemiológicas de Pacientes Hospitalizados por Infarto Agudo do Miocárdio em Belém do Pará.

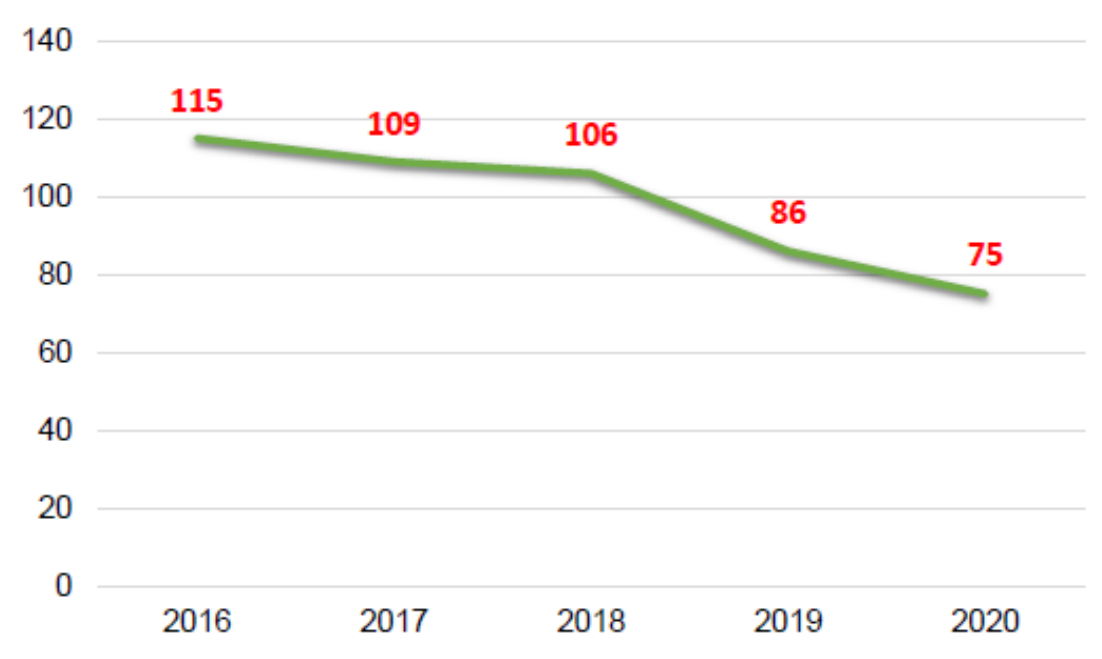

Figura 3: Representação do número de óbitos por IAM durante o período de 2016 a 2020, em Belém do Pará.

Fonte: Ministério da Saúde. SIH/SUS. 2021.

Apesar de ter sido notada uma queda brusca nos valores absolutos de óbitos por IAM, sobretudo nas hospitalizações de 2020 , não é possível associar esta redução à melhoria do manejo pela equipe multiprofissional e nem a melhoria da infraestrutura hospitalar intensiva local, necessariamente. Similar à analogia das internações, a queda do número de internações ao longo do ano de 2020 pode ter influenciado no menor número de registros de óbitos por IAM. ${ }^{12,22}$

\section{CONSIDERAÇŌES FINAIS}

O presente estudo revelou um total de 5.854 pacientes internados por IAM em Belém do Pará entre 2016 a 2020. Dentre as características epidemiológicas avaliadas, notou-se que a maioria foram do sexo masculino $(70,8 \%)$, pardos $(80 \%)$ e idosos $(62,29 \%)$. Esses valores concordam com grande parte da literatura, visto que colocam as maiores prevalências de IAM concentradas nos indivíduos do sexo masculino, negros/pardos e idosos.

Apesar de ter sido notado uma queda vultuosa nos números de óbitos dos pacientes hospitalizados por IAM, sobretudo em 2020, não foi possível associar esse resultado a melhorias do manejo intensivo e da infraestrutura hospitalar. Ao que parece, semelhante a outros estudos, a queda do número de óbitos durante o momento supracitado teve 
DOI: 10.18606/2318-1419/amazonia.sci.health.v9n1p69-78 Revista Amazônia Science \& Health
ALVAREZ, ACC. REZENDE, RWS. SOUSA, EJS.

Características Epidemiológicas de Pacientes Hospitalizados por Infarto Agudo do Miocárdio em Belém do Pará.

influência direta da pandemia do SARS-CoV-2/COVID-19. A maioria das internações, em centros intensivos durante $\mathrm{o}$ ano de 2020, esteve direcionada aos pacientes com a síndrome respiratória influenciando, dessa forma, a uma diminuição do número de internações por outros motivos pela escassez de leitos.

Ademais, nota-se a importância da atenção primária à saúde na capital paraense em suas ações de prevenção e promoção de saúde relacionadas às DCV's. As investidas na atenção básica auxiliam na redução das prevalências de DCV's, bem como na diminuição de gastos futuros com terapêuticas especializadas. Outrossim, investimentos em leitos de terapia intensiva se fazem necessários, haja vista que outras demandas emergenciais ainda acontecem em meio a pandemia e estas requerem a devida intervenção.

\section{REFERÉNCIAS}

1. Musher DM, Abers MS, Corrales-Medina, VF. Acute infection and myocardial infarction. N. Engl. J. Med. 2019; 380: 171-176.

2. Lippi G, Sanchis-Gomar F, Cervellin G. Chest pain, dyspnea and other symptoms in patients with type 1 and 2 myocardial infarction. A literature review. Int. J. Cardiol. 2016.

3. Townsend N, Wilson L, Bhatnagar P, Wickramasinghe K, Rayner M, Nichols M. Cardiovascular disease in Europe: Epidemiological update 2016. Eur. Heart J. 2016; 37: 3232-3245.

4. Rossello X, Lobo-Gonzalez M, Ibanez B. Editor's Choice- Pathophysiology and therapy of myocardial ischaemia/reperfusion syndrome. Eur Heart J Acute Cardiovasc Care. 2019; 8:443-456.

5. Smilowitz NR, Weiss MC, Mauricio R, Mahajan AM, Dugan KE, Devanabanda A, et al. Provoking conditions, management and outcomes of type 2 myocardial infarction and myocardial necrosis. Int. J. Cardiol. 2016.

6. Januzzi JL, Sandoval Y. The many faces of type 2 myocardial infarction. J. Am. Coll. Cardiol. 2017; 70:1569-1572.

7. Nascimento BR, Brant LCC, Oliveira GMM, Malachias MVB, Reis GMA, Teixeira RA, et al. Epidemiologia das Doenças Cardiovasculares em Países de Língua Portuguesa: Dados do "Global Burden of Disease", 1990 a 2016. Arq Bras Cardiol. 2018; 110(6):500511.

8. Vaz DWN, Rezende RWS, Barros TVL, Atta JFM, Filho LCCS, Miranda TLKS. Descrição epidemiológica de pacientes hospitalizados com IAM no Estado do Pará (de 2015 a 2019). Rev. Bra. Edu. Saúde. 2020; 10(1): 45-50.

9. Amsterdam EA, Wenger NK, Brindis RG, Casey DE, Ganiats TG, Holmes DR, et al. 2014 AHA/ACC guideline for the management of patients with non-st-elevation acute 
DOI: 10.18606/2318-1419/amazonia.sci.health.v9n1p69-78 Revista Amazônia Science \& Health
ALVAREZ, ACC. REZENDE, RWS. SOUSA, EJS.

Características Epidemiológicas de Pacientes Hospitalizados por Infarto Agudo do Miocárdio em Belém do Pará.

coronary syndromes: A report of the American college of cardiology/American heart association task force on practice guidelines. 2014; 130 :e344-e426.

10. Ro M, Patrono C, Collet JP, Mueller C, Valgimigli M, Andreotti F, et al. 2015 ESC Guidelines for themanagement of acute coronary syndromes in patients presenting without persistent st-segment elevation: Task force for the management of acute coronary syndromes in patients presenting without persistent ST-segment elevation of the European Society of Cardiology (ESC). Eur. Heart J. 2016; (37): 267-315.

11. Knuuti J, Wijns W, Achenbach S, Agewall S, Barbato E, Bax JJ, et al. 2019 ESC guidelines for the diagnosis and management of chronic coronary syndromes. Eur. Heart J. 2020; 41: 407-477.

12. Mascarenhas MDM, Batista FMA, Rodrigues MTP, Barbosa OAA, Barros VC. Ocorrência simultânea de COVID-19 e dengue: o que os dados revelam?. Cad. Saúde Pública. 2020; 36(6):e00126520.

13. Liu PP, Blet A, Smyth D, Li H. The Science Underlying COVID-19: Implications for the cardiovascular system. Eur. Heart J. 2020.

14. Madjid M, Safavi-Naeini P, Solomon SD, Vardeny O. Potential effects of coronaviruses on the cardiovascular system: A review. JAMA Cardiol. 2020.

15. Driggin E, Madhavan MV, Bikdeli B, Chuich T, Laracy J, Bondi-Zoccai G, et al. Cardiovascular considerations for patients, health care workers, and health systems during the coronavirus disease 2019 (COVID-19) pandemic. J. Am. Coll. Cardiol. 2020.

16. Claro RM, Santos MAS, Oliveira TP, Pereira CA, Szwarcwald CL, Malta DC. Consumo de alimentos não saudáveis relacionados a doenças crônicas não transmissíveis no Brasil: Pesquisa Nacional de Saúde, 2013. Epidemiol. Serv. Saúde. 2015; 24(2): 257-265.

17. Miranda $\mathrm{CH}$, Aimoli US. Competência Clínica no Manejo do Infarto Agudo do Miocárdio com Supradesnível do Segmento ST por Médico Recém-Formado Candidato à Residência Médica. Arq Bras Cardiol. 2020; 114(1):35-44.

18. Varenne O, Cook S, Sideris G, Kedev S, Cuisset T, Carrie D, et al. Drug-eluting stents in elderly patients with coronary artery disease (SENIOR): a randomised single-blind trial. Lancet. 2018; 391:41-50.

19- Safdar B, Spatz ES, Dreyer RP, Beltrame JF, Lichtman JH, Spertus JA, et al. Presentation, clinical profile, and prognosis of young patients with myocardial infarction with nonobstructive coronary arteries (MINOCA): results from the VIRGO study. J Am Heart Asso. 2018; 7:e009174.

20. Almeida-Santos MA. et al. Análise espacial e tendências de mortalidade associada a doenças hipertensivas nos estados e regiões do brasil entre 2010 e 2014. International Journal of Cardiovascular Sciences. 2018; 31(3): 250-257.

21. Polanczyk CA. Epidemiologia das Doenças Cardiovasculares no Brasil: A Verdade Escondida nos Números. Arq Bras Cardiol. 2020; 115(2):161-162. 
DOI: 10.18606/2318-1419/amazonia.sci.health.v9n1p69-78 Revista Amazônia Science \& Health 2021, Vol. 9, № 1
ALVAREZ, ACC. REZENDE, RWS. SOUSA, EJS.

Características Epidemiológicas de Pacientes Hospitalizados por Infarto Agudo do Miocárdio em Belém do Pará.

22. Ibáñez B. Myocardial infarction in times of COVID-19 Infartos en tiempos de la COVID19. Rev Esp Cardiol. 2020; 73(12):975-977. 